\title{
Identidad institucional de las organizaciones del Tercer Sector
}

\author{
Isabel de la Torre Prados \\ Universidad Autónoma de Madrid \\ isabel.torre@uam.es
}

Resumen: El objetivo del artículo es ofrecer una aproximación descriptiva a los rasgos distintivos de las entidades del Tercer Sector, tanto desde el punto de vista de sus características organizativas como de su gobierno y gestión, considerando que estas tres líneas de análisis están condicionadas por el reconocimiento institucional de las funciones sociales que desempeñan el conjunto plural y beterogéneo de sus entidades, entendidas como organizaciones no lucrativas cuyos rasgos básicos son el carácter privado, el principio de no distribución de beneficios, la movilización de recursos voluntarios, la estructura organizativa formal y la capacidad de autonomía y gobierno.

Palabras clave: Organigramas horizontales, modelo de gestión, gobierno transparente, identidad institucional, imagen pública.

Abstract: This article's objective is to offer a descriptive approximation to the distinctive features of the Third Sector's entities, through their organizational characteristics, government and management, considering that these three lines of analysis are conditioned by the institutional acknowledgement of the social functions that are carried out by the plural and heterogeneous collection of their entities, understood as charity organizations whose main features are privacy, principle of no profit distribution, voluntary resources mobilization, formal organizational structure and capacity of autonomy and governance.

Keywords: horizontal organizational charts, management model, transparent government, institutional identity, public image. 


\section{Introducción}

Los principales fundamentos teóricos que se establecen en torno al Tercer Sector hacen referencia, en un primer momento, a las nociones de participación, sociedad civil y acción colectiva en el contexto de una sociedad compleja en la que ha alcanzado un apreciable grado de desarrollo el Estado de bienestar. Estos conceptos están relacionados con teorías explicativas políticas y sociales en las que el ejercicio de la ciudadanía comparte presencia en la escena pública con movimientos sociales, partidos políticos y sindicatos en el contexto de cambio experimentado por el Estado de bienestar en nuestra sociedad global.

La eclosión en las últimas tres décadas de distintas formas de participación ciudadana a través de las entidades del Tercer Sector discurre paralelamente a los cambios producidos en el Estado de bienestar en el nuevo escenario de la globalización, cuando, a principios de la década de 1980, se cuestiona la viabilidad del modelo vigente debido a la aplicación de la contención del gasto social en las políticas sociales desarrolladas por el Estado de bienestar en los años sesenta y setenta, período expansivo de crecimiento y modernización de la sociedad española (Rodríguez Cabrero, 2003).

El nuevo contexto se caracteriza por el cambio de roles del sector público y del sector privado, y también de las relaciones que se establecen entre uno y otro. El Tercer Sector va conformándose y desarrollando su principal actividad en la provisión de bienestar social colectivo y en el fomento de nuevas formas de participación social. Estas dos tendencias, formuladas por Anheier (2005), implican dos dimensiones:

a) Las entidades sin ánimo de lucro como aprovisionadoras de servicios sociales

Responde a una tendencia de carácter neoliberal, en la que las entidades se identifican con actuaciones de colaboración con el gobierno en la gestión del bienestar social. Anheier destaca el papel de estas entidades como «contratistas que prestan servicios que son retribuidos, en parte, por el gobierno» (Anheier, 2005), por lo que su presencia garantiza la extensión de la cobertura del Estado, sobre todo a aquellos colectivos afectados por problemáticas sociales específicas.

La perspectiva neoliberal entiende la descentralización del Estado en el ámbito de los servicios sociales como una forma de organización de la sociedad civil que resulta operativa a efectos de resolver situaciones sociales mediante la intervención del Tercer Sector. Las entidades de este sector responden así a una lógica de descentralización del Estado que opera bajo la fórmula de que menos intervención implica menos burocracia, más flexibilidad y mayor eficiencia (Anheier, 2005). 
b) Nuevas formas de acción y participación de la sociedad civil

El Tercer Sector se presenta como un instrumento de cohesión social ante la creciente pérdida de participación en organizaciones sociales tradicionales como la Iglesia, los sindicatos o los partidos políticos. El tejido social se reactiva a partir de las nuevas propuestas que ofrecen las organizaciones no lucrativas, en las que, bajo un interés común, se expresan nuevas formas de asociación y movimiento de personas, con especial protagonismo del voluntariado.

El marco de la participación, asociado a los movimientos sociales, surgidos en las décadas de los setenta y los ochenta, se modifica para dar paso a las formas voluntarias de participación social, que canalizan las entidades no lucrativas, y que también van evolucionando a la vez que se producen cambios en la propia organización y en la gestión de estas entidades. La evolución también implica una continua confrontación entre dos perspectivas: una perspectiva que resalta la importancia de estas nuevas formas de participación social, a las que confiere una capacidad creativa de ciudadanía activa (Zurdo Alaguero, 2003), y, por otro lado, una perspectiva que identifica la necesidad de una administración eficaz del trabajo de la organización que garantice su supervivencia, con vistas a la consecución de los fines para los que se creó.

Ambas dimensiones favorecen el reconocimiento institucional de las organizaciones no lucrativas y la exigencia de mejorar los sistemas de gobierno y gestión, ya que, al ser aprovisionadoras de servicios, las entidades no lucrativas se integran en el flujo de recepción de financiación para los proyectos de intervención social por parte de la Administración estatal y del sector privado, ya sean particulares o empresas, lo que implica un mayor grado de formalización de sus estructuras organizativas y el cumplimiento de una serie de requisitos que garanticen la consecución de sus objetivos y la consolidación de su imagen pública, aspecto esencial del Tercer Sector sobre el que pivota su modelo de gestión y gobierno.

Los requisitos que acompañan a la formalización de las organizaciones redundan en perseguir una mejora de la gestión y administración de sus actividades, en impulsar la participación de las personas voluntarias que apoyan y trabajan en ellas, y en ofrecer una información transparente de los resultados conseguidos al conjunto de la sociedad. Todos ellos incluidos en unas estructuras organizativas suficientemente flexibles que permiten la plena integración de los elementos formales e informales y facilitan el desarrollo de su actividad desde la participación activa de sus integrantes (Herrera y Ayuso, 2009). 


\section{Características organizativas de las entidades del Tercer Sector}

Las entidades no lucrativas comprenden una relación amplia de objetivos y una casuística variada de orígenes a partir de los cuales sus formas de actuación e intervención sociales se estructuran y orientan. Son organizaciones «caracterizadas por el rasgo común de su orientación al bienestar general, por su capacidad de respuesta a motivaciones de acción colectiva y por representar los valores de un modelo de convivencia social en el marco de una sociedad civil organizada» (De la Torre, 2005).

Un rasgo diferenciador de estas organizaciones es la doble presencia de aportación voluntaria personal (de conocimientos y experiencias, contribución económica, objetos y utensilios) y de aportación profesional, entendida como una aportación cualificada que tiene especial importancia en determinadas entidades.

Las actuaciones de las entidades no lucrativas fomentan la participación social en dos sentidos:

- Como participantes receptores de una intervención específica que atiende demandas sociales que surgen en la sociedad y a las que trata de dar respuesta cada organización en su sector.

- Como grupo de personas voluntarias y de profesionales dispuestos a colaborar y trabajar en la línea de dicha actuación (Pérez-Díaz y López Novo, 2003).

A la hora de abordar la estructura organizativa del Tercer Sector, los aspectos más visibles a considerar son su tamaño, su sector de actividad, su evolución —entendida en sentido positivo o crecimiento y en sentido negativo o desaparición- y sus relaciones con el sector público y el sector privado a través de actuaciones conjuntas y de la confluencia de objetivos complementarios (Zurdo Alaguero, 2007).

La interrelación de los tres sectores refleja posibles alianzas que refuerzan sus objetivos:

- El Estado: a través del Tercer Sector canaliza la provisión de servicios sociales para la población y cuenta con entidades que fomentan la participación cívica.

- El sector privado: a través de personas individuales y de empresas, que con sus aportaciones o patrocinios impulsan y colaboran en proyectos e intervenciones sociales con una orientación altruista y solidaria, concretada en ocasiones en un compromiso social formalizado en el compromiso empresarial de la responsabilidad social corporativa.

- El Tercer Sector: dependiendo de los fines de cada organización, gestiona las contribuciones económicas e invierte en la consecución de sus objetivos, que son, fundamentalmente, la acción e intervención sociales en sectores y poblaciones afectados por una problemática social o por nuevas formas de exclusión 
colectiva, en torno a los cuales se genera un movimiento hacia la intervención social que combina participación voluntaria y trabajo profesional.

Por otra parte, la tendencia a la formalización no siempre cristaliza en estructuras organizativas reconocidas, sino que se puede manifestar de formas diversas, según cuáles sean la intención y los medios disponibles para satisfacer las diferentes demandas sociales. Las diferentes fases del proceso pueden identificarse con tres modalidades de estructuras organizativas (Ruiz Olabuenaga, 2007):

- Agrupaciones sociales, que resultan de la interacción y coordinación de las personas, pero sin que conlleve una racionalización de procesos encaminados a conseguir objetivos comunes planteados.

- Organizaciones formales, cuando existen estructuras de coordinación en torno a unos objetivos ordenados según una jerarquía que canaliza de forma eficaz la gestión de los recursos y las actuaciones en favor de la consecución de los fines de la organización.

- Organizaciones informales, surgidas entre los propios miembros de una organización formal, ya sean explícitas o no reconocidas.

El Tercer Sector, por lo tanto, ofrece un tejido organizacional muy flexible en el que se integran las organizaciones formales, que pueden tener su propia regulación jurídica (asociaciones, fundaciones, organizaciones no gubernamentales); las agrupaciones con presencia y actuación puntuales que no adoptan una organización permanente, y los componentes informales de interrelación social presentes en toda organización. El grado de organización refleja la propia evolución del sector, que abarca desde el nivel de los movimientos sociales y las formas de participación no estructuradas hasta las organizaciones de acción colectiva con fines de intervención, transformación social e incidencia básicas con un elevado nivel de coordinación y administración. En todo caso, la flexibilidad de sus formas organizativas no significa renunciar a conseguir un mínimo de permanencia, una estructura interna y unas normas regladas (Izquieta Etulain, 2008).

A la hora de especificar el proceso de configuración de las estructuras organizativas de las entidades del Tercer Sector hay que ampliar la relación de variables que influyen en la forma organizativa adoptada, tales como:

- Finalidad de la organización y sector de intervención social.

- Marco normativo.

+ Tamaño de la organización.

- Ámbito territorial de actuación.

- Orígenes y evolución histórica de cada organización.

- Presencia conjunta de profesionales remunerados y de personas voluntarias. 
A) Por lo que se refiere a la finalidad y al área de intervención, Naciones Unidas presenta una amplia clasificación comúnmente aceptada en trece grandes áreas (Naciones Unidas, 2003): cultura, deporte y ocio; educación e investigación; salud; servicios sociales; medio ambiente; desarrollo y vivienda; derechos civiles y políticos; intermediarios filantrópicos y promoción del voluntariado; actividades y programas internacionales; religión; negocios y actividades profesionales; otras no clasificadas.

En relación con cada área sectorial, se distingue una amplia clasificación de diferentes formas organizativas:

- Organizaciones proveedoras de servicios sociales: como hospitales, instituciones educativas, centros de cuidado y asistencia social, grupos de protección del medio ambiente.

- Organizaciones no gubernamentales* para la promoción del desarrollo económico y la reducción de la pobreza.

- Organizaciones artísticas y culturales: entre las que se incluyen museos, centros de representación escénica, conjuntos musicales, sociedades históricas y literarias, etc.

- Asociaciones deportivas: orientadas al entrenamiento, el mantenimiento físico o la preparación de competiciones.

- Grupos de apoyo: dedicados a la promoción de los derechos civiles y sociales, y a la incidencia política en relación con intereses de grupos socialmente vulnerables.

- Fundaciones $\$$ entidades que cuentan con activos y donaciones que les permiten subvencionar proyectos de otras organizaciones o financiar sus propios proyectos y programas.

- Asociaciones locales $\leftleftarrows$ en las que los miembros pertenecen a un vecindario, comunidad, pueblo, etc., y que se crean con el objetivo de ofrecer servicios en su propio entorno.

- Partidos políticos: entidades que tienen la capacidad de representación de los ciudadanos en los asuntos de interés público, para hacerlos partícipes de la vida política.

- Asociaciones culturales: cuyas actividades están centradas en el ocio y el tiempo libre.

- Uniones corporativas empresariales $y / o$ sindicales: para la promoción y defensa de intereses laborales y profesionales.

- Organizaciones religiosas $\$$ inspiradas en creencias y valores solidarios y altruistas con orientación espiritual. 
Las áreas de intervención y sus diferentes organizaciones muestran una pluralidad de estructuras organizativas de mayor o menor complejidad.

B) Respecto a la normativa jurídica, entre las entidades no lucrativas se distinguen las asociaciones, las fundaciones y las organizaciones no gubernamentales. En el caso de España, las entidades más numerosas y las que más recursos económicos y humanos movilizan (García Delgado et alii, 2009) son las asociaciones y las fundaciones. Los tres tipos de entidades presentan una regulación específica:

- Las asociaciones se rigen por la Ley Orgánica 1/2002, de 22 de marzo, reguladora del Derecho de Asociación, ${ }^{1}$ en la que se establecen los aspectos relacionados con la creación, el funcionamiento y la declaración de utilidad pública de las asociaciones si cumplen determinados requisitos (De Lorenzo García, 2005).

- Las fundaciones se regulan a partir de la Ley 49/2002, de 23 de diciembre, de Régimen Fiscal de las Entidades sin Fines Lucrativos y de los Incentivos Fiscales al Mecenazgo, que determina los tres elementos básicos de este tipo de entidades: ausencia de fines lucrativos, tributación en el impuesto sobre sociedades, y aplicación del régimen de tributos locales (que depende de la autonomía en la que tengan su ámbito de actuación). Según sus objetivos fundacionales y las actividades que lleven a cabo, pueden clasificarse como asistenciales, culturales, docentes o laborales.

- Las organizaciones no gubernamentales de desarrollo (ONGD) tienen una naturaleza jurídica específica, según la Ley 23/1998, de 7 de julio, de Cooperación Internacional para el Desarrollo, artículo 32, en el que se especifica como objeto expreso «la realización de actividades relacionadas con los principios y objetivos de la cooperación internacional para el desarrollo». ${ }^{2}$

C) El tamaño de la organización tiene una relación directa con el grado de formalización de la estructura organizativa y con la búsqueda de permanencia, ya que un determinado tamaño implica el establecimiento de normas regladas para asegurar su funcionamiento y la adopción de procedimientos cualificados de gestión para garantizar la provisión de recursos procedentes del sector público y del sector privado.

D) El tamaño de la organización está relacionado con el ámbito territorial de actuación de las entidades y supone una estructura organizativa más compleja con un núcleo central y distintas organizaciones dependientes. En la práctica se establece una diversidad de niveles en relación con los procedimientos de gestión y organización interna, así como en relación con la capacidad de extensión de su marco de actuación e

1 Ley Orgánica 1/2002, de 22 de marzo, reguladora del Derecho de Asociación. Disponible en www.boe.es. 2 Para obtener financiación de carácter público, es requisito indispensable que la ONGD conste en el Registro Oficial, que está adscrito a la Agencia Española de Cooperación Internacional para el Desarrollo (AECID). 
intervención sociales. Las organizaciones pueden, por lo tanto, estructurarse a nivel local, regional o nacional. Dentro de esta clasificación, asimismo, se puede distinguir entre las organizaciones que cuentan con sedes en diversos territorios y las que las tienen dentro del propio país, lo que se traduce en una mayor cantidad de recursos con los que abordar el diseño y la ejecución de sus proyectos.

E) A lo largo de su desarrollo histórico, las acciones en pro del bienestar social han estado inspiradas en el rasgo común de la ausencia de lucro, aunque el origen y la evolución históricos de cada organización están basados en motivaciones de carácter religioso, altruista o solidario. El acto fundacional de las entidades del Tercer Sector tiene especial importancia a la hora de mantener viva la misión originaria de cada entidad como núcleo central de la organización y base de su reputación social; ambos elementos constituyen el núcleo esencial de su capital social y contribuyen a la proyección simbólica de la imagen pública del Tercer Sector.

F) Finalmente, el elemento diferenciador más característico en el funcionamiento de las organizaciones del sector no lucrativo es la aportación conjunta del trabajo profesional y del trabajo voluntario, que condiciona la articulación funcional interna de las estructuras organizativas y de sus modelos de gestión. Además, la presencia de personas voluntarias en la actuación de estas entidades, al representar físicamente los valores del altruismo y la solidaridad, refuerza la esencia de su identidad institucional y consolida la imagen simbólica del sector no lucrativo, entendida como proceso de identificación compartida con otros y en favor de otros, frente a otras formas de identificación basadas en la exclusión y el enfrentamiento con otros.

Las variables mencionadas (finalidad y objetivos de cada entidad, sectores de actividad, normativa jurídica, tamaño, ámbito territorial, orígenes y evolución histórica, y presencia del voluntariado) influyen de modo diferente en la configuración de la estructura organizativa de cada entidad del Tercer Sector, y suponen retos importantes a la hora de examinar los procesos de gestión y dirección e incluso de proponer modelos homogéneos de gestión y dirección. Además, a la dificultad de ofrecer unas directrices de gestión válidas para el conjunto de entidades que conforman el Tercer Sector, se añade el factor de cambio y transformación que el propio sector experimenta y que responde a la complejidad de los elementos que lo conforman y a su nexo de unión con la sociedad civil. Por todo ello, es necesario adoptar una perspectiva dinámica a la hora de examinar la realidad organizativa de las entidades del Tercer Sector para detectar el grado de influencia interna de las variables conformadoras y las formas de expresión asociativa de la participación ciudadana.

Las estructuras organizativas de las entidades del Tercer Sector tienden a concretarse en organigramas flexibles y horizontales, en los que se refleja que la consecución de los objetivos de eficacia y eficiencia debe llevarse a cabo en forma de aprendizaje abierto 
y en equipo Senger (1995), con la finalidad de reforzar el reconocimiento social de su identidad institucional.

\section{Gestión y gobierno de las organizaciones del Tercer Sector}

La pluralidad de características organizativas, anteriormente descritas, incide en la heterogeneidad de las estructuras organizacionales y está en consonancia con los parámetros de gestión de las entidades no lucrativas, generalmente muy alejados del desarrollo alcanzado por la gestión de las organizaciones empresariales, que se refleja en la abundante literatura sobre administración y dirección empresariales.

Durante mucho tiempo, el mundo de la solidaridad ha mantenido una actitud marcada por la indiferencia hacia lo que podríamos denominar las lógicas empresariales de administración y gestión. Una de las principales razones de esta situación está vinculada a la tradicional tendencia de los directivos de estas organizaciones a dar por descontada la «bondad intrínseca» de sus actividades. La mera existencia de la asociación o fundación es vista como un hecho positivo, como un indicador de «buen trabajo», y se deja en un segundo plano la necesidad de alcanzar unos objetivos concretos y de dar cuenta puntual de los resultados.

Sin embargo, en los últimos años se ha ido imponiendo la necesidad de rendir cuentas a la sociedad en relación con los recursos financieros que reciben las entidades, vía Administración pública y/o por iniciativa del sector privado, principalmente por la necesidad de aplicar rigor al control de los recursos públicos y por la presión social de transparencia en su gestión, de tal modo que la gestión de estas entidades ha pasado progresivamente del planteamiento tradicional de estar basada en el principio de hacer el bien a los otros a un modo de operar más racional desde el punto de vista de la gestión y de hacer bien la actividad que se orienta al bien de los otros.

En este nuevo escenario es obligado preguntarse si la necesidad de que las organizaciones del Tercer Sector adopten modelos de gestión que se centren en la calidad de las prestaciones realizadas y en los niveles de éxito de sus diversos componentes supone adoptar modelos de gestión más afines a la gestión empresarial basados en la eficacia y en la eficiencia, o si, por el contrario, las entidades del Tercer Sector en su pluralidad organizativa son capaces de desarrollar modelos especificos de gestión en los que se garantice la calidad de la acción externa y de la gestión interna.

Por otra parte, desde los años noventa se aprecian líneas de convergencia en la gestión de las entidades no lucrativas y en la gestión de las empresas, debido a la difusión alcanzada por las prácticas de responsabilidad social corporativa como resultado 
de aplicar un modelo de gestión basado en los principios de sostenibilidad y respeto ambiental y en los llamados grupos de interés: empleados, proveedores, accionistas, clientes y comunidad local. Este último grupo de interés, el de la comunidad local, ha desarrollado distintas modalidades de acción social promovidas por las empresas con el apoyo y la mediación de entidades no lucrativas.

En el proceso de convergencia hacia la profesionalización de la gestión de las entidades no lucrativas se vienen aplicando prácticas instrumentales de gestión empresarial, como ocurre con los diagnósticos basados en el análisis DAFO, ${ }^{3}$ acrónimo que sintetiza la identificación de las debilidades, las amenazas, las fortalezas y las oportunidades, representadas en una matriz que facilita la elaboración de planes de mejora y el correspondiente fortalecimiento institucional de cada entidad. Frente al modelo DAFO, adaptado de las organizaciones lucrativas, existe otra propuesta de autoconocimiento más afín a las características organizativas y de gestión de las entidades no lucrativas; se trata del modelo de gestión de triple corona (Aragón-Correa, 2004), que consiste en diferenciar tres niveles de gestión: el primero, denominado corona central, en el que prima la misión o finalidad última de la organización como elemento inspirador y esencial de la gestión; el segundo nivel, que es el denominado corona intermedia, se refiere a los objetivos definidos y jerarquizados para conseguir la finalidad última de la organización, siempre que se orienten a facilitar la mutua comprensión entre todos los miembros de la organización en sus diferentes niveles y a otorgar credibilidad a la entidad frente a terceros; el tercer nivel o corona externa se refiere a los programas de actuación que contribuyen a lograr los objetivos establecidos en el marco de la cultura participativa vigente en estas entidades, siguiendo el criterio de aunar flexibilidad de propuestas y compromiso común, una vez fijada la jerarquía de actuaciones para alcanzar los objetivos establecidos.

\subsection{La gestión de las organizaciones del Tercer Sector}

Cualquiera que sea el modelo de gestión adoptado, las entidades no lucrativas suelen coincidir en unas formas específicas de realizar su gestión, que se manifiestan en cinco grandes apartados:

a) Planificación y organización, cuya principal peculiaridad es determinar unos objetivos cuyo seguimiento y resultado no están sujetos exclusivamente a criterios económicos y obligan, por lo tanto, a enriquecer el tradicional esquema de inputs y outputs en la generación y distribución de productos y servicios.

3 La Fundación Luis Vives realiza desde hace tres años un proyecto, cofinanciado con el Fondo Social Europeo, para elaborar diagnósticos individualizados de las entidades solicitantes y seleccionadas en cada convocatoria anual. http://www.fundacionluisvives.org/servicios/apoyo_entidades/diagnosticos_organizativos_ong/48408/index.html 
b) Gobierno, entendido como defensa de la misión establecida por los promotores de la entidad, que debe transformarse en objetivos y conseguir el apropiado cumplimiento de éstos.

c) Recursos económicos, procedentes de ayudas y conciertos con el sector público y de aportaciones del sector privado de carácter finalista, es decir, que no contemplan la existencia de excedentes ni su posible capitalización.

d) Separación entre donante y receptor, que en términos empresariales suele materializarse en la relación comercial entre proveedor y cliente, y supone una forma de revalidar resultados a través del grado de satisfacción del cliente.

e) Aportación de trabajo voluntario, que se suma a la actividad profesional remunerada y requiere adoptar modelos de autoridad basados en el liderazgo moral y en el manejo apropiado de procedimientos fundados en la gestión participativa eficaz y eficiente.

En cada uno de los apartados mencionados pueden existir riesgos que afectan tanto a la viabilidad de las actuaciones de intervención social como a la permanencia del núcleo de valores que nutren la misión y garantizan la identidad de estas organizaciones (Christensen y Molin, 1995). Hay dos apartados en los que los riesgos cobran especial importancia:

\subsection{La gestión de los recursos económico-financieros}

La particularidad de las fuentes de financiación de las entidades del Tercer Sector está estrechamente ligada a las características organizativas, a la tipología de las actividades que desarrolla y a las funciones sociales que toda organización del Tercer Sector desempeña. Las diferencias con las empresas de mercado y con las agencias públicas son notables y se manifiestan en la forma mixta que presentan las entradas de recursos financieros en las organizaciones del Tercer Sector, ya sea con aportaciones de particulares y empresas, con subvenciones y ayudas procedentes del sector público, o, en algunos casos, con ganancias procedentes de alguna actividad mercantil mediadora como sorteos, rastrillos, fiestas y eventos organizados con este fin.

Las principales fuentes de financiación de las organizaciones del Tercer Sector son las siguientes:

a) Entradas derivadas del sector público.

b) Entradas cuyo origen son donaciones de particulares y empresas.

c) Entradas derivadas de las cuotas asociativas.

d) Entradas procedentes de actividades mercantiles mediadoras. 
a) La financiación pública constituye una de las entradas más extendidas entre las organizaciones del Tercer Sector. Internamente, esta fuente de financiación se articula en dos secciones: financiación directa y financiación indirecta. En el ámbito de la financiación directa se encuentran los dos principales instrumentos de soporte y apoyo a disposición del sector público para promover y promocionar las organizaciones del Tercer Sector: el sistema de las contribuciones y de las subvenciones y el sistema de las convenciones mediante convenios o contratos. La modalidad de financiación indirecta se desdobla también en dos tipos de medidas: las primeras proceden del conjunto de disposiciones encaminadas a conferir un estatus de exención (total o parcial) a la actividad y al patrimonio de la organización; las segundas se refieren a la posibilidad de deducir del propio ingreso imponible, por parte de aquellos que ofrecen las contribuciones económicas, toda o parte de la suma donada.

b) El segundo tipo de financiación es el que se refiere a las entradas derivadas de donaciones del sector privado y posiblemente constituye la fuente de financiación más característica de estas organizaciones, aquella que de manera más explícita las distingue de las empresas de mercado y de las agencias públicas. Las contribuciones económicas englobadas en la categoría de donaciones tienen un doble origen:

- Donaciones de particulares (ciudadanos contribuyentes y familias).

- Donaciones de empresas y otras instituciones (fundaciones, empresas comerciales).

c) El tercer tipo de entradas financieras está constituido por las cuotas asociativas y las contribuciones procedentes de los miembros de la organización. Se trata de una forma tradicional de financiación que no requiere la utilización de técnicas y mecanismos de gestión particularmente sofisticados. Más bien se debe desarrollar una política de marketing interno, es decir, de atención a los propios miembros, que deben ser considerados y tratados como clientes de la organización. La capacidad de escucha (atención a las sugerencias procedentes de los socios) y la voluntad para poner en práctica las acciones prometidas, además de la adopción de una modalidad de gestión participativa, son elementos que refuerzan el clima motivacional de la organización y, con ello, el sentido de pertenencia de los miembros individuales.

d) El cuarto tipo de financiación son las entradas procedentes de actividades mercantiles mediadoras que se originan en dos tipos de actividades:

- Las entradas derivadas de la realización de actividades comerciales propiamente dichas, que deben tener un carácter secundario en la finalidad de la organización $y$, si se quieren beneficiar de las exenciones fiscales eventualmente previstas, deben ser completamente invertidas en la organización con el fin de mejorar la capacidad de consecución de los fines vigentes en los estatutos. Un ejemplo representativo es el sorteo extraordinario de la Cruz Roja. 
- En algunas entidades se contempla también un eventual recargo monetario por determinados servicios establecidos para la consecución del propio fin asociativo y regulado en sus estatutos. Un ejemplo puede ser el recargo sobre la utilización de determinados servicios especiales en una asociación de ayuda a enfermos de Alzheimer.

Si se exceptúa la fuente de financiación procedente de las cuotas de los miembros, que puede ofrecer un cierto margen de seguridad a los ingresos de las entidades compatible con posibles devoluciones y bajas, el resto de las vías de financiación requieren esfuerzos de gestión notables que exigen un grado de cualificación especializada por parte de sus responsables y la adopción de procedimientos de captación y seguimiento de los recursos. Aun así, las fuentes de financiación ofrecen un equilibrio inestable que afecta a la planificación de las actividades y a la consecución de los objetivos programados.

\subsection{La gestión de las personas}

El otro gran apartado relacionado con las entidades no lucrativas es el de la gestión de las personas que integran las organizaciones, ya que constituye uno de los aspectos cruciales y una de las tareas principales de la dirección de estas entidades, pues las organizaciones que proporcionan servicios (y las del Tercer Sector están entre éstas) exigen una alta intensidad de trabajo y una determinada fracción de recursos aportados para cada unidad generada de output. Además, las organizaciones voluntarias deben conciliar la presencia de profesionales y voluntarios, de aquí que los especialistas en la materia (Jeavons, 1993; Brudney, 1993; Young et alii, 1993) dividan las personas que trabajan en las organizaciones del Tercer Sector en dos grupos diferenciados con necesidades muy diversas y, a veces, opuestas: personal retribuido y personal voluntario.

- Respecto al personal retribuido, los puntos cruciales son el momento de la contratación (selección) y el establecimiento de un sistema de incentivos, pecuniarios o no, coherente con la misión de la organización. Otros momentos importantes son los relacionados con la promoción y los planes de carrera.

- En cuanto al personal voluntario, las fases más importantes son: el reclutamiento, la permanencia en la organización, la asignación a una tarea determinada, los aspectos motivacionales y el reconocimiento interno. Las estrategias para el reclutamiento de voluntarios pueden basarse en una pluralidad compleja de motivaciones que van desde el interés personal (autoestima, reputación, carrera, crecimiento personal...) hasta motivos altruistas (bienestar de la comunidad, valores de solidaridad y caritativos, beneficios sociales...). La gestión de las personas voluntarias se regula desde 1996 por la Ley del Voluntariado de 
ámbito estatal. También en los últimos años casi todas las comunidades autónomas han establecido normas legales sobre el voluntariado; en algunos casos se trata de leyes aprobadas por sus parlamentos respectivos y, en otros casos, de decretos administrativos dentro de su ámbito territorial en desarrollo de sus competencias. En términos generales, se trata de normas que pretenden «promover y facilitar la participación solidaria de los ciudadanos en las actuaciones de voluntariado, en el seno de organizaciones sin ánimo de lucro públicas o privadas» (Ley del Voluntariado 6/1996). En todo caso, como regla general hay que tener presente «los deberes y derechos de los que son portadores los voluntarios, con arreglo a los cuales deben aplicarse las normas legales existentes o las que tenga establecidas la organización y que deben ser conocidas y aceptadas por los voluntarios. Tampoco hemos de olvidar el compromiso temporal que adquieren y que, en la medida de lo posible, debería formalizarse por escrito», según sugiere Gutiérrez Resa (1997).

La complejidad de la gestión y la dirección de personas en las organizaciones del Tercer Sector se concreta en dos direcciones principales: competir por la adquisición de personal en el mercado de trabajo y, en su caso, atraer también al personal voluntario. Una vez contratados o movilizados, establecer incentivos ad hoc para mantener su motivación.

Algunas características de estas organizaciones, y a pesar de las particulares limitaciones de los recursos disponibles, pueden favorecer la atracción de personal cualificado respecto a las empresas de mercado; entre ellas cabe mencionar:

a) Una atención y un compromiso constantes con la calidad.

b) La imagen de la organización y su reputación como institución solidaria y agencia distribuidora de bienes de utilidad pública.

c) La capacidad de proporcionar beneficios no pecuniarios compensatorios en lugar de recompensas financieras.

Un aspecto complementario a considerar como reflejo de la confluencia entre la gestión empresarial y la gestión de las entidades no lucrativas es el que se refiere al voluntariado corporativo, que es una modalidad de aportación voluntaria en proyectos y actuaciones de intervención social que, impulsada por las empresas entre sus empleados para implicarles en modelos de gestión basados en la responsabilidad social corporativa, ha alcanzado una importante difusión desde finales de los años noventa.

Tras este recorrido, no resulta aventurado llegar a la siguiente conclusiónः garantizar los recursos a través de las vías disponibles de financiación y facilitar la gestión de las personas y de las relaciones interpersonales son los dos grandes retos planteados en la gestión de las entidades del Tercer Sector, y ambos exigen una manera apropiada de entender y aplicar el gobierno y la dirección. 


\subsection{El gobierno de la organización}

Una de las mayores diferencias entre las organizaciones del Tercer Sector y las empresas de mercado viene dada por el rol desempeñado por el órgano al que corresponde el gobierno de la organización (las denominaciones más comunes son las de consejo de administración, comité de gestión, consejo de dirección, comité ejecutivo) y la función de aprobación en la toma de decisiones atribuida a la Asamblea, que tiene una máxima capacidad en el caso de las asociaciones. En general, la acción de gobierno está representada en una doble función: a) externa, referida a las relaciones que la entidad no lucrativa mantiene con el entorno de la organización; $b$ ) interna, manifestada en las relaciones del órgano de gobierno con los sectores internos de la organización.

La función externa engloba el conjunto de medidas encaminadas a establecer la legitimación de la organización en la comunidad y a reforzar su capacidad de actuación, especialmente aquellas medidas que tienen como fin atraer los recursos económicos. La función interna implica coordinar las directrices de gobierno con las actividades de los profesionales y, en su caso, de las personas voluntarias con el objetivo de garantizar los servicios que la organización presta a la comunidad. El desempeño de ambas funciones requiere lograr un pacto de confianza mutua entre el órgano de gobierno, los directivos y todas las personas que integran la organización, diferenciándose dos líneas paralelas de autoridad: la primera, del consejo de administración al personal operativo, y la segunda, de los profesionales a los voluntarios.

Los principales asuntos tratados y discutidos en la literatura sobre los enfoques adoptados en la dirección de las entidades no lucrativas pueden resumirse en los siguientes apartados (Young et alii, 1993):

1. El gobierno de la organización. Se someten a examen los mecanismos a través de los que las organizaciones del Tercer Sector ponen a punto sus objetivos a largo plazo a la vez que conservan su integridad (se analizan las actividades del consejo de administración o comité de gestión y la actuación de los directivos y mandos intermedios). El principio básico a seguir es el de transparencia a nivel interno y en la comunicación externa.

2. Dirección ejecutiva. En este apartado se agrupan los estudios sobre los procedimientos y las medidas instrumentales que los directivos de estas organizaciones consideran operativos para el desarrollo de las políticas y las orientaciones generales. Las figuras clave son: el consejo, el director, los cuadros intermedios $y$ todos aquellos que tienen responsabilidades en la gestión de la organización.

3. Gestión de los recursos financieros. El problema de la disponibilidad económicofinanciera que caracteriza a estas organizaciones, unido al carácter peculiar de sus fuentes de financiación, obliga al desarrollo de técnicas de gestión y de re- 
cogida de fondos muy específicas. La peculiaridad de las medidas a emplear deriva de la necesidad de encontrar un equilibrio entre las entradas, ya que éstas presentan exigencias de gestión no siempre conciliables: financiación pública, donaciones y contribuciones privadas, cuotas asociativas e ingresos procedentes de actividades mercantiles puntuales. En este caso, el principio de transparencia se aplica a la rendición de cuentas.

4. Gestión de las personas. En función de las características estructurales de estas organizaciones, que las configuran como organizaciones de alta intensidad de trabajo, la gestión del personal resulta ser uno de los aspectos cruciales de la administración, incluyendo los procedimientos que facilitan la gestión del personal contratado, del personal voluntario y de los colaboradores externos.

5. Gestión estratégica del cambio. Una considerable serie de presiones externas influyen de manera decisiva en las entidades no lucrativas, ya que les afectan notablemente las influencias institucionales y funcionales del ambiente organizativo en que actúan. En concreto, estas organizaciones están subordinadas a los cambios del contexto legal-administrativo, a las mutaciones del clima político-social, a los desafíos derivados del cambio social y a las innovaciones procedentes del campo metodológico y profesional.

Desde una perspectiva personal, los máximos responsables de las entidades no lucrativas cumplen dos importantes cometidos de la dirección: por una parte, el dirigente desempeña un rol emprendedor, en cuanto iniciador de nuevos programas o a través del desarrollo de nuevos recursos; por otra, sobre él recae la responsabilidad de la gestión del personal, con la exigencia de mantener los presupuestos de la misión establecida en el origen fundacional, adaptándola a la evolución experimentada por la organización.

En suma, la coherencia entre el modelo de gestión y la forma de gobierno de las entidades no lucrativas es condición indispensable para garantizar la permanencia de la propia entidad; tan importante es mantener el objetivo de su misión fundacional como la eficacia y calidad de su actuación y el reconocimiento de su función de interés general por el conjunto de la sociedad. Desde esta perspectiva, la búsqueda de la excelencia en la gestión y el gobierno de las entidades no lucrativas refuerza su identidad institucional y favorece la reputación social del Tercer Sector y su imagen pública. 


\section{Las funciones del Tercer Sector y su reconocimiento social}

Las entidades del Tercer Sector desarrollan actuaciones dirigidas a determinados grupos de población que son reconocidas como de interés general por el conjunto de la población y están vinculadas a diferentes funciones sociales. El reconocimiento de estas funciones por parte de la sociedad genera un efecto de retroalimentación entre las entidades como agentes sociales y la sociedad que recibe los beneficios de su actuación, a través de la acción e intervención sociales de las organizaciones, pero también a través del fomento de la participación ciudadana y las distintas vías de vinculación: voluntariado, profesional remunerado, miembro activo, miembro cotizante y vinculación puntual.

Entre las funciones sociales que se reconocen al Tercer Sector destacan las actuaciones que definen su núcleo central, es decir, las derivadas de su acción social.

\section{a) Función vertebradora de cohesión social}

Las entidades del Tercer Sector actúan de forma complementaria al Estado en la provisión de bienestar social a la población, en especial a colectivos socialmente excluidos o vulnerables. A través de las organizaciones no lucrativas, las administraciones públicas gestionan esta forma de colaboración Tercer Sector-Estado mediante la financiación de proyectos de intervención social directamente gestionados por estas organizaciones.

Por otra parte, la mayor demanda de financiación para las actuaciones de intervención social supone la incorporación del sector privado, tanto de particulares como de empresas. Por ejemplo, el Tercer Sector puede canalizar algunas iniciativas empresariales que, desde la responsabilidad social corporativa, aportan recursos para satisfacer demandas de la sociedad que se cubren a través de la actuación de las entidades no lucrativas.

La capacidad de articular aportaciones privadas y recursos públicos cuya gestión se orienta a satisfacer demandas sociales de grupos minoritarios - que son percibidas por el conjunto de la población como si fueran de interés general- supone reconocer una función vertebradora en la cohesión social al Tercer Sector. ${ }^{4}$

\footnotetext{
4 Ejemplo: la Asociación de Síndrome de Down Castellón, junto con la fundación del mismo nombre, se presenta en su página web con el objetivo de trabajar por la «promoción y realización de cuantas actividades contribuyan a la mejora de la calidad de vida de las personas con síndrome de Down o con otro tipo de discapacidad psíquica». En el apartado de colaboraciones aparecen entidades del ámbito privado-empresarial y del institucional-público que apoyan económicamente estos proyectos. http://www.downcastellon.com
} 


\section{b) Función simbólica}

El Tercer Sector no limita su actuación a la provisión de servicios sociales, sino que cumple, además, una función simbólica que la transciende: erigirse en referencia para el ejercicio de la ciudadanía responsable y la práctica de la solidaridad, lo que tiene reflejo en las colaboraciones personales, ya sean como simpatizantes, socios contribuyentes, voluntarios o profesionales, en las organizaciones no lucrativas. El incremento del número de estas organizaciones y la respuesta asociativa de la población española (De la Torre y López Ruiz, 2010) pueden tomarse como un indicador de la fortaleza del tejido social y de una mayor capacidad de actuación e influencia de la sociedad civil organizada.

En este sentido, la función simbólica del Tercer Sector refuerza la posibilidad expresiva de las personas que se identifican con los valores representados en estas organizaciones, lo que produce un impacto en el orden político, bien sea cuestionándolo o bien sea mejorándolo. ${ }^{5}$

\section{c) Función política}

Como se ha apuntado en la función simbólica, las organizaciones de intervención social tienen un importante papel en la expresión de valores, siendo al mismo tiempo instrumentos de testimonio y agencias distribuidoras de servicios. También contribuyen a la promoción de los valores cívicos, de los derechos de ciudadanía y de la participación de las personas en la vida pública. Pero la dimensión más definitiva de esta función política radica en la verdadera capacidad de transformación social que se puede llevar a cabo desde el Tercer Sector detectando problemas, dándoles visibilidad social y proponiendo soluciones.

Esta función social se produce cuando las demandas sociales se elevan hasta alcanzar un espacio en la agenda política, tratando de conseguir una respuesta más directa y comprometida por parte de la Administración pública. Las entidades no lucrativas tienen de esta forma, en el campo de actuación de la esfera pública, un espacio donde los temas sociales en que trabajan deben aparecer y adquirir consistencia, de modo que lleguen a trasladarse al ámbito político. Para ello, desarrollan estrategias de sensibilización sobre sus fines y objetivos, dando visibilidad a sus organizaciones y a los fines que persiguen.

5 Dos ejemplos: SOS Racismo lleva a cabo diferentes tipos de acciones y actividades de sensibilización y denuncia. Una de estas actividades ha sido la realización del Manual de prevención de incidentes racistas en las aulas, dirigido al profesorado de educación primaria y secundaria (http://www.sosracismo.org/).

Fundación Hazloposible: entre las iniciativas que lleva a cabo, se encuentra el programa de voluntariado corporativo dirigido a empresas. La asistencia técnica de esta fundación (http://www.hazloposible.org/) se oferta a través del programa de voluntariado corporativo más adecuado a las características de la empresa y de la plantilla; el alineamiento del programa de voluntariado corporativo con la estrategia de RSE de la empresa; la creación del portal de voluntariado, y su difusión entre los empleados. 
Las estrategias pueden llevarse a cabo a partir de las acciones de una organización en particular, o que sea un grupo de organizaciones que comparten los mismos objetivos las que realicen acciones conjuntas, con el fin de conseguir desde un espacio reconocido en la opinión pública y mediática hasta una mayor incidencia y presión en el nivel político. ${ }^{6}$

\section{d) Función de mejora y calidad de los servicios}

Las organizaciones no lucrativas desarrollan su trabajo en áreas de intervención y acción sociales, y disponen, por lo tanto, de un conocimiento acumulado sobre la forma más adecuada de llevar a cabo esas intervenciones. Este saber instrumental influye en el proceso de provisión de los servicios sociales y en la propia evolución del Estado del bienestar, que tiende a delegar cada vez más la tarea intervención en los sectores de población socialmente vulnerables, pues las actuaciones promovidas por las entidades voluntarias, al estar libres de los procedimientos burocráticos, son unas respuestas rápidas y efectivas a las demandas de los grupos minoritarios (Montagut, 2003).

También, al trabajar en el propio contexto de estos grupos, hay más posibilidades de adelantarse a los problemas que surgen y avanzar propuestas para mitigarlos o erradicarlos de forma eficaz. Esta dinámica de trabajo es un continuo aprendizaje para el personal de las entidades y, a la larga, influye en la profesionalización del sector, lo que asegura la adecuada aplicación del proceso de trabajo y gestión organizativos: desde la identificación de problemas hasta el diseño del proyecto y su aplicación.

La experiencia del trabajo y el contacto diario con colectivos que tienen problemas sociales determinados son la base del conocimiento profesional con la que cuentan las organizaciones. Este conocimiento ratifica sus competencias en el terreno en el que desarrollan sus actividades, y las valida como interlocutoras entre los grupos de población con los que trabajan y las instituciones, públicas y privadas, para desarrollar y aplicar proyectos que cada vez requieren un mayor conocimiento especializado de los temas y una experiencia más sólida sobre los grupos de población en los que se quiere intervenir. $^{7}$

\footnotetext{
6 Es ilustrativo el ejemplo de Greenpeace, que consigue una cobertura informativa de gran alcance en cada una de sus acciones de protesta. Otro ejemplo es el que se refiere a la Ley Orgánica 1/2004, de 28 de diciembre, de Medidas de Protección Integral contra la Violencia de Género, que es el resultado de todo un trabajo anterior de presión política por parte de una red de organizaciones y asociaciones feministas que exigían su promulgación (http://www.redfeminista.org/laley.asp).

7 Ejemplo: para la elaboración del II Plan de Acción contra la Explotación Sexual de la Infancia y la Adolescencia, el Ministerio de Trabajo y Asuntos Sociales acudió a Save the Children para organizar una mesa de trabajo nacional, en la que participaron directamente ONG y profesionales relacionados con la infancia procedentes de distintas CCAA; de esta mesa se derivaron múltiples propuestas que han sido consideradas y recogidas en el II Plan de Acción contra la Explotación Sexual de la Infancia y la Adolescencia. Observatorio de la Infancia, 2006-2009. Secretaría de Estado de Servicios Sociales, Familias y Discapacidad. Ministerio de Trabajo y Asuntos Sociales. http://www.mtas.es/SGAS/ FamiliaInfanc/infancia/PlanesInformes/ExplotSex.pdf
} 
Por otra parte, la necesidad de tener personal especializado en las entidades no lucrativas exige promover su formación y su cualificación, de este modo las entidades no lucrativas garantizan ante la sociedad su capacidad técnica de gestión de los servicios que prestan a la población y la correcta transmisión de las demandas de grupos sociales minoritarios.

\section{e) Función de orientación y asesoramiento}

El número de entidades no lucrativas constituidas ha experimentado en los últimos años un notable crecimiento. Una de las consecuencias de este crecimiento han sido los cambios que afectan a los procedimientos y a la profesionalización de la gestión y dirección de las organizaciones. La proliferación de entidades supone una mayor competitividad en la búsqueda de financiación para los proyectos, una competencia que, en muchos casos, está formalizada y en otros, además de los requisitos establecidos, depende en gran medida del reconocimiento de una gestión eficaz y de un gobierno transparente, reflejado en la reputación obtenida en los sectores públicos y privados.

El proceso de profesionalización de las organizaciones no lucrativas se ha entendido como una adaptación de las formas de gestión identificadas con el sector privado y la administración de empresas. No sólo cambian los modos de proceder en el diseño y la aplicación de proyectos, sino que se integran otros elementos organizacionales no directamente relacionados con la intervención social, como son la realización de auditorías, la gestión de los recursos humanos y las actuaciones de comunicación e información de las actividades fuera de la organización.

Estos cambios internos han tenido un efecto en el campo de actuación de las propias organizaciones, y en los servicios que prestan, al propiciar la aparición de entidades que ofrecen servicios de apoyo y asistencia técnica para la aplicación de procedimientos de mejora de la gestión y del funcionamiento de las entidades no lucrativas, y que también se dirigen a otras instituciones públicas y privadas con el objetivo de ofrecerles orientación sobre cómo establecer una relación de colaboración entre las organizaciones no lucrativas y la Administración, las empresas y los particulares. Asimismo, surge la figura de los observatorios como un elemento clave a la hora de presentar una información sistematizada sobre los movimientos y las actuaciones de las organizaciones. ${ }^{8}$

\footnotetext{
8 Ejemplos: la Fundación Lealtad es una institución sin ánimo de lucro que tiene como uno de sus objetivos «ofrecer a particulares y empresas información independiente, objetiva, homogénea y gratuita sobre las ONG para ayudarles a decidir con qué ONG colaborar y orientarles para hacer un seguimiento de sus donaciones». Más información: http:// www.fundacionlealtad.org/

El Observatorio del Voluntariado se crea como centro de investigación para el estudio del voluntariado y su objetivo es: «Conocer la realidad del voluntariado y sensibilizar y comprometer a la sociedad con el movimiento voluntario, la participación ciudadana y los valores que éstos representan, mediante la visibilización y el estudio de la evolución del movimiento voluntario organizado del tercer sector de acción social». Una contribución de especial relevancia es el Plan Estratégico del Tercer Sector (2005-2010). http://www.plataformavoluntariado.org/web/observatory/index
} 


\section{Conclusiones}

El examen de las características organizativas de las entidades no lucrativas, especialmente de su rasgo más significativo, que es la presencia de personal voluntario, muestra la pluralidad de sus estructuras organizativas y la dificultad de proponer modelos de gestión y gobierno para organizaciones tan heterogéneas. Aun así, la creciente exigencia de profesionalidad en la administración y gestión de los recursos y de garantía en la calidad del servicio ha conllevado la aparición de numerosas prácticas de gestión y gobierno y de suficientes referencias en la literatura especializada para orientar la toma de decisiones y el seguimiento de los objetivos de las entidades no lucrativas.

Las orientaciones en la gestión se centran en tres grandes apartados: planificación y organización, gestión de los recursos económico-financieros y gestión de las personas. Por lo que se refiere al gobierno de estas entidades, basado en el principio de transparencia a nivel interno y externo, hay tres apartados de especial consideración. Los dos primeros son de carácter externo: la rendición de cuentas del ejercicio presupuestario programado y la información periódica de las actividades realizadas a través de las memorias, los informes y las páginas web. El tercer apartado es de carácter interno: la información participativa y democrática de los procedimientos seguidos en el ejercicio de las actividades.

La adecuada gestión y el buen gobierno de las entidades no lucrativas persiguen la permanencia de las organizaciones y el reconocimiento de las funciones sociales que realizan confirmando la identidad institucional de cada entidad, entendida como el reconocimiento social de su orientación al bienestar general desde los valores del altruismo y la solidaridad. La coherencia entre la gestión y el gobierno de las entidades no lucrativas contribuye a reforzar la imagen pública del Tercer Sector y mejora su visibilidad social y la valoración positiva de sus actuaciones y servicios en el seno de una sociedad civil organizada (De la Torre, 2007).

\section{Bibliografía}

Anheier, H. K. (2005). «¿Qué clase del Tercer Sector? ¿Qué tipo de sociedad? Efectos de las políticas gubernamentales en las organizaciones sin fines de lucro», en J. L. García Delgado (coord.). La Economía Social en España. Madrid: Fundación ONCE.

Aragón-Correa, J. A. et alii (2004)+ «Gobierno y Gestión de las entidades del Tercer Sector», en J. L. García Delgado (coord.). La Economía Social en España. Madrid: Fundación ONCE. 
Brudney, J. L. (1993). "Strengthening Volunteer Administration through Continuing Education and Research», en D. R. Young Young; R. M. Hollister; V. M. Hodgkinson et alii (1993). Governing, Leading and Managing Nonprofit Organizations. San Francisco: Jossey-Bass Publishers.

Christensen, S. y Molin, J. (1995). «Organizational Adaptation to Conflicting and Shifting Institutional and Technical Enviroments», en R. Scotr y S. ChrisTEnsen (eds.). The Institutional Construction of Organizations. California: SAGE Publications.

De la Torre, I. y López Ruiz, J. A. (2010). Imagen pública del Tercer Sector y participación asociativa en España: 2002-2009. Madrid: Fundación ONCE (edición en curso).

De la Torre, I. (2007). «Imagen pública del Tercer Sector en España». CIRIEC, 57.

De la Torre, I. (2005). «Tercer Sector y participación ciudadana en España. Opiniones y Actitudes». Reis, 51.

De Lorenzo García, R. (2005). «Tipología jurídica de las organizaciones. Un repaso a los distintos tipos de personas jurídicas y su legislación aplicable». Revista Española del Tercer Sector, 1, oct.-dic 2005.

García Delgado, J. L. et alii (2009). Las cuentas de la economía social. Magnitudes y financiación del Tercer Sector en España. Madrid: Editorial Civitas Thomson.

Gutiérrez Resa, A. (1997). Acción Social No Gubernamental. Análisis y reflexiones sobre las organizaciones voluntarias. Valencia: Tirant lo Blanch.

Herrera Gómez, M.y Ayuso Sánchez, L. (2009). «Las asociaciones sociales, una realidad a la búsqueda de conceptuación y visualización», Reis, 126.

Izquieta Etulain, J. L+ (2008). «Las organizaciones no lucrativas. Naturaleza y dinámica actual», en J. A. VAlero Matas (coord.) (2008). Las instituciones y organizaciones sociales. Madrid: Editorial Pirámide.

Jeavons, T. H. (1993). «The Role of Values: Management in Religiosus Organizations», en D. R. Young Young; R. M. Hollister; V. M. Hodgkinson et alii (1993). Governing, Leading and Managing Nonprofit Organizations. San Francisco: Jossey-Bass Publishers.

Ley Orgánica 1/2002, de 22 de marzo, reguladora del Derecho de Asociación. Disponible en www.boe.es.

Montagut, T. (2003). «Voluntariado y cambio social», en T. Montagut (coord.) Voluntariado: la lógica de la ciudadanía. Madrid: Ariel Sociología.

Naciones Unidas (2003). Handbook of National Accounting: Handbook on Non-Profit Institutions in the System of National Accounts. New York: United nation Publication. Disponible en http://unstats.un.org/unsd/publication/SeriesF/SeriesF_91E.pdf 
Pérez Díaz, V. (2005). «Sociedad civil y Tercer Sector», en J. L. García Delgado (coord.). La economía social en España. Madrid: Fundación ONCE.

Pérez-Díaz, V. y López Novo, J. (2003). El Tercer Sector Social en España. Madrid: Ministerio de Trabajo y Asuntos Sociales.

Rodríguez Cabrero, G. (coord.) (2003). Las entidades voluntarias de acción social en España. Informe General. Madrid: Fundación FOESSA.

Ruiz Olabuenaga, J. I. (2007). Sociología de las organizaciones complejas. Bilbao: Universidad de Deusto.

Senger, P. (1995). La quinta disciplina en la práctica. Estrategias y herramientas para construir la organización abierta al aprendizaje. Barcelona: Editorial Granica.

Young, D. R. ; Hollister, R. M. y Hodgrinson, V. M. et alii (1993). Governing, Leading and Managing Nonprofit Organizations. San Francisco: Jossey-Bass Publishers.

Zurdo Alaguero, A. (2007). «La dimensión corporativa del Tercer Sector». Revista Internacional de Sociología (RIS), LXV (47) mayo-agosto.

Zurdo Alaguero, Á. (2003). «Voluntariado y estructura social: funciones sociales y límites», en G. Rodríguez Cabrero (coord.). Las entidades voluntarias de acción social en España. Informe General. Madrid: Fundación FOESSA. 